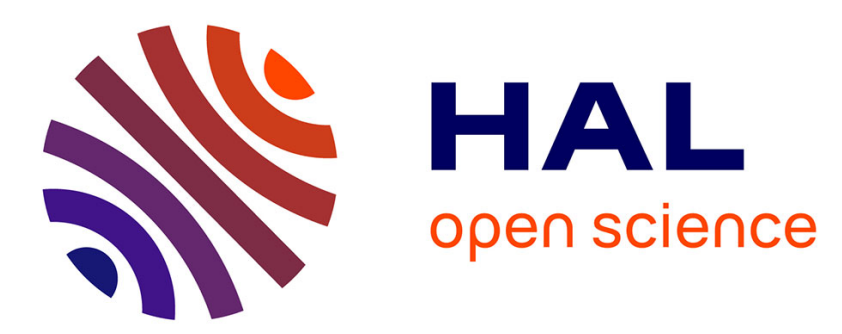

\title{
Mesurer la diversité et l'impact des actions de lutte contre la discrimination: état des lieux et pistes de réflexion
}

Hédia Zannad

\section{To cite this version:}

Hédia Zannad. Mesurer la diversité et l'impact des actions de lutte contre la discrimination : état des lieux et pistes de réflexion. Humanisme et entreprise, 2009, n²95, pp.30-40. hal-00568665

\section{HAL Id: hal-00568665 \\ https://hal.science/hal-00568665}

Submitted on 16 Mar 2012

HAL is a multi-disciplinary open access archive for the deposit and dissemination of scientific research documents, whether they are published or not. The documents may come from teaching and research institutions in France or abroad, or from public or private research centers.
L'archive ouverte pluridisciplinaire HAL, est destinée au dépôt et à la diffusion de documents scientifiques de niveau recherche, publiés ou non, émanant des établissements d'enseignement et de recherche français ou étrangers, des laboratoires publics ou privés. 


\title{
Mesurer la diversité et de lutte contre la discrimination : état des lieux et pistes de réflexion
}

\author{
Hédia Zannad \\ Professeur Associée \\ Rouen Business School \\ Bd André Siegfrid, 76825 Mont Saint Aignan \\ hzn@ rouenbs.fr
}

\section{Résumé}

La lutte contre la discrimination et la promotion de la diversité sont devenues des enjeux forts pour les entreprises mais leur mesure pose encore des problèmes d'ordre opérationnel, communicationnel et stratégique que cet article cherche à éclairer. Sur la base d'une revue de littérature et d'une étude de terrain -essentiellement qualitative-, quatre parties sont abordées : nous cherchons, tout d'abord, à comprendre les notions et les enjeux propres à la diversité et à la discrimination ; nous montrons, ensuite, qu'au-delà des seules dimensions techniques, la diversité renvoie à des aspects tactiques et pédagogiques clé; nous éclairons, dans une troisième temps, les pratiques d'une quinzaine d'entreprises sur le sujet; nous ébauchons, enfin, des pistes d'amélioration des indicateurs qu'il s'agit de sélectionner.

\section{Mots clé}

Discrimination, Diversité, Egalité de traitement, Egalité des chances, Mesure.

\begin{abstract}
Companies are increasingly struggling against discrimination and promoting diversity, but their measurement raises operational, strategic and communicational problems that we want to highlight. On the basis of a literature review and a qualitative field research, we develop four parts in the paper: first, we differentiate anti-discrimination and diversity; then, we demonstrate that diversity comprises technical but also tactic and pedagogical key dimensions; third, we report the practices of fifteen companies on the subject; finally, we suggest ideas to select pertinent measurement boards.
\end{abstract}

\section{Key words}

Discrimination, Diversity, Equality of treatment, Equality of opportunity, Measurement. 


\section{Mesurer la diversité et de lutte contre la discrimination : état des lieux et pistes de réflexion}

Si les entreprises françaises ont longtemps été dans une relative indifférence face à la diversité et la lutte contre les discriminations, le sujet prend une importance croissante. Plusieurs facteurs expliquent cette mouvance, parmi lesquelles: la loi du 11 février 2005 sur le handicap a entraîné la mise en place de politiques volontaristes en matière de recrutement des personnes en situation de handicap; la création de la HALDE et sa médiatisation ont provoqué une prise de conscience dans les entreprises de l'étendue de la discrimination et des risques pénaux engendrés par elle pour l'entreprise elle-même et pour ses collaborateurs ; la condamnation pour discrimination raciale de l'Oréal et d'Adecco et sa large médiatisation ont mis en avant les risques qu'encourent les entreprise en termes d'image et de réputation; la pénurie de main d'œuvre dans certains secteurs et certaines fonctions et le départ en retraite des baby boomers ont poussé les entreprises à mettre en questions leurs politique de sourcing et à s'ouvrir à des profils jusqu'à là ignorés ; la création, fin 2008, du Label Diversité par l'ANDRH et l'AFNOR; la loi du 17 décembre 2008 de financement de la sécurité sociale pour 2009, qui a instauré l'obligation pour les entreprises de mettre en place un plan d'actions sur l'emploi des seniors sous peine d'une amende de $1 \%$ de la masse salariale, etc.

Notre question de recherche pourrait se formuler ainsi : quels sont les enjeux sous-jacents à la mesure de la diversité ? Plus précisément, quelle(s) nature(s) revêt la construction et l'utilisation d'indicateurs en la matière : opérationnelle, communicationnelle, stratégique...? Notre propos n'est pas de proposer des indicateurs parmi lesquels les entreprises pourraient choisir celles qui leur semblent les plus pertinentes; ce serait faire fi du travail de construction des indicateurs en fonction des objectifs, contraintes, contexte et culture spécifiques à chaque organisation. En effet, ainsi que l'affirment Feisthammel et Massot (2005, p. 177), «La sélection et la hiérarchisation des critères de performance qu'on affecte à un indicateur trouvent leurs sources dans l'état organique, politique, technique ou stratégique de l'organisation. » Il s'agira donc plutôt de mettre en avant, en fonction de notre revue de littérature (sur la diversité d'une part, les indicateurs et les tableaux de bord, d'autre part) et de notre étude de terrain, des définitions, éclairages conceptuels, principes, typologies, et exemples d'indicateurs afin de faciliter le travail des managers de la diversité à progresser dans leur projet. 
Pour répondre à notre question de recherche, nous avons animé quatre tables rondes avec les responsables Diversité d'une quinzaine d'entreprises - L'Oréal, Adecco, Accor, BNP-Paribas, La Poste, Véolia Environnement, CNP, SFR, Groupe Keyrus, Schneider Electric, Mornay- sur le sujet de la mesure de la diversité. L'ensemble des échanges effectués durant ces tables rondes et présentations a été retranscrit sous forme de compte-rendu détaillé. Parallèlement à ces moments collectifs, des questionnaires fermés ont été administrés à l'ensemble de ces entreprises afin de recueillir des données précises sur les objectifs poursuivis à travers la mesure de la diversité et les indicateurs en usage. Par la suite, des entretiens semi-directifs ont été menés avec l'ensemble de ces acteurs ; après avoir été intégralement retranscris, ils ont fait l'objet d'une analyse de contenu. Nous avons également effectué des entretiens auprès d'entreprises n'ayant pas participé à la Commission de l'AFMD sur les indicateurs afin d'accroître la représentativité de notre échantillon (Sanofi-Aventis, Orange, Bristol-Myers Squibb). Bien entendu, toutes les données secondaires qui nous ont été offertes durant ces entretiens (chartes, rapports de développement durable, brochures internes...) ont été exploitées. L'ensemble de ces captures de données visait à établir un bilan en matière d'indicateurs de la diversité (objectifs, interrogations, état des lieux, difficultés rencontrées) mais aussi plus largement à comprendre les enjeux et pratiques de ces entreprises en matière de diversité et de discrimination. Ce triple matériau est donc de nature mixte: données primaires et secondaires, qualitatives et quantitatives.

\section{Préambule : quelques éclaircissements sur la mesure de la diversité}

\section{Diversité et discrimination : les deux faces d'une même pièce ?}

Les textes officiels nous permettent de définir précisément ce qu'est la discrimination en France et dénombrent 18 critères de discrimination: "Constitue une discrimination toute distinction opérée entre les personnes physiques à raison de leur origine, de leur sexe, de leur situation de famille, de leur grossesse, de leur apparence physique, de leur patronyme, de leur état de santé, de leur handicap, de leurs caractéristiques génétiques, de leurs moeurs, de leur orientation sexuelle, de leur âge, de leurs opinions politiques, de leurs activités syndicales, de leur appartenance ou de leur non-appartenance, vraie ou supposée, à une ethnie, une nation, une race ou une religion déterminée. »(Code pénal).

Identifier les dimensions de la diversité en revanche est complexe. Car, contrairement à la discrimination, aucun texte juridique ne définit la diversité. Jusqu'où aller dans les 
caractéristiques à retenir ? Choisir une définition restrictive -genre, âge, origine ethnique, etc. - risque de diluer chacune des caractéristiques dans un ensemble général qu'est la diversité. Opter pour une définition large nous rappelle que chaque être est dissemblable et peut donc apporter sa différence propre, sa singularité. Ce qu'on peut dire en revanche est que les entreprises engagées dans une démarche active d'accroissement de la diversité travaillent le plus souvent sur quatre grandes dimensions de la diversité : égalité professionnelle (genre), âge, handicap et origine ethnique.

De fait, il s'agit de sortir de la confusion qui est souvent faite entre mesure de la diversité et mesure de la discrimination, de la part de l'ensemble des acteurs clé. Or, si la mesure de la discrimination renvoie à une norme et un objet clairement établi, ce n'est pas le cas de la diversité. En effet, mesurer la discrimination vise à se mettre en conformité avec la loi en rectifiant certaines pratiques et processus opératoires (logique juridique). Mesurer la diversité vise à davantage refléter les proportions des différents groupes de personnes d'une société à l'intérieur d'une organisation (logique politique) mais que pourrait être un «bon» ou un « mauvais » niveau de diversité ? Nul ne le sait.

\section{Genre, âge, handicap, origine ethnique : des problématiques analogues ?}

Si le genre, l'âge, le handicap et l'origine ethniques sont les quatre variables qui figurent le plus souvent dans l'enveloppe diversité, force est de constater qu'elles ne renvoient nullement, d'après nous, aux mêmes problématiques - en France du moins- même si elles sont toutes traversées par la même réalité : l'existence de préjugés fortement ancrés. Nous avons fait figurer ci-dessous ces divergences d'enjeux (liste non exhaustive) :

\begin{tabular}{|c|c|}
\hline Genre & $\begin{array}{l}\text { - Comment encourager l'investissement de la sphère familiale par les } \\
\text { hommes ? } \\
\text { - Comment réduire l'autocensure effectuée par les femmes pour l'accession à } \\
\text { des postes à responsabilité ? } \\
\text { - Comment faire évoluer le modèle dominant de leadership (mobilité } \\
\text { géographique, disponibilité inconditionnelle, etc.) }\end{array}$ \\
\hline Age & $\begin{array}{l}\text { - Comment permettre aux « seniors » insuffisamment qualifiés d'accéder au } \\
\text { marché du travail (rappelons que la majorité des Comex est composés de } \\
\text { seniors) } \\
\text { - Comment redéfinir la notion de hauts potentiels afin de ne pas y inclure une } \\
\text { limite d'âge? } \\
\text { - Comment valoriser l'apport des seniors dans des systèmes de tutorat? }\end{array}$ \\
\hline Handicap & $\begin{array}{l}\text { - Comment favoriser l'auto-déclaration des travailleurs ayant un handicap non } \\
\text { visible (afin, notamment de réduire les cotisations à l'Agefiph)? } \\
\text { - Comment accroitre le nombre de candidatures (avec un niveau de }\end{array}$ \\
\hline
\end{tabular}




\begin{tabular}{|l|l|}
\hline & $\begin{array}{l}\text { qualification élevé) ? } \\
\text { - Comment faire évoluer les stéréotypes courants (qui poussent généralement à } \\
\text { se représenter un handicap lourd)? }\end{array}$ \\
\hline \multirow{3}{*}{$\begin{array}{l}\text { Origines } \\
\text { ethniques }\end{array}$} & $\begin{array}{l}\text { Comment réduire la discrimination effectuée à l'égard des personnes de } \\
\text { couleur ou ayant un patronyme à consonance étrangère tout au long de leur } \\
\text { parcours ? }\end{array}$ \\
& $\begin{array}{l}\text { Comment désamorcer le repli communautaire dans les équipes de travail très } \\
\text { diversifiées ? }\end{array}$ \\
\hline
\end{tabular}

Dès lors, ignorer ces divergences profondes et construire un système de pilotage antidiscriminatoire ou pro-diversité indifférencié selon les dimensions retenues risque d'aboutir à une certaine inefficacité.

En outre, une même dimension de la diversité peut avoir des échos forts différents d'un pays à l'autre. C'est notamment le cas, nous le savons, de l'origine ethnique aux USA et européens (nous y reviendrons plus spécifiquement) mais c'est également le cas, par exemple, de la situation des femmes en France et au Royaume-Uni. En effet, ces deux pays enregistrent des taux d'emploi similaires (60\% pour le premier, 67\% pour le second), mais les femmes disposent de structures publiques les aidant à concilier vie professionnelle et familiale tout en ne bénéficiant pas d'un traitement égalitariste sur le lieu de travail en France alors que la situation est strictement inversée pour les femmes du Royaume-Uni (étude McKinsey, 2007).

\section{Indicateurs et tableaux de bord : de la technique à la stratégie}

\section{Indicateurs et tableaux de bord : définition, rôle et critères de performance}

L'indicateur renvoie à un lien de signification déterminé entre un support de présentation et un élément constitutif du déroulement réel d'un évènement. Il se situe dans un monde virtuel, entre le monde réel de l'organisation et le monde mental de l'utilisateur (Fernandez, 2005). Il contient les éléments permettant d'établir ce lien original (certes, indirect car dépendant des phases intermédiaires) et, par conséquent, de mesurer l'effet des actions entreprises. Il a pour rôle de mesurer une situation et de provoquer une réaction (comprenant celle de ne rien faire) du décideur en fonction de l'objectif visé. Précisons qu'un objectif imprécis comporte des inconvénients majeurs : effets nuls en terme d'action et de communication, charge de travail inutile, perte de crédibilité de la DRH (Taïeb, 1996). 
Trois types d'indicateurs peuvent être distingués : les indicateurs d'alerte, d'équilibration et d'anticipation. Les indicateurs d'alerte signalent un état anormal du système, exigeant une intervention rapide. Les indicateurs d'équilibration font état du système et de sa progression vers l'objectif, et peuvent susciter des actions correctives en cas de dérive. Les indicateurs d'anticipation fournissent des informations de prévision plus larges pouvant induire des changements de stratégie ou d'objectif. En résumé, ces trois types d'indicateurs jouent respectivement un rôle d'alarme, de suivi et de prévision. Les mesures peuvent être effectuées soit en valeur brute sur une échelle ouverte ou fermée, soit en écart par rapport à une ou plusieurs valeurs de référence, qu'il s'agisse d'une butée ou d'un standard.

Une seconde catégorisation peut être utilement proposée: les indicateurs de moyen et les indicateurs de résultat. A titre d'exemple, le nombre de travailleurs handicapés, de seniors ou de femmes à des postes à responsabilité dans une entreprise -indicateurs de résultats- peut être accru par une politique de recrutement attractive, des mesures favorisant l'équilibre vie professionnelle/vie privée ou l'investissement dans des formations permettant la déconstruction de préjugés -indicateurs de moyens. Néanmoins, cette distinction n'est pas toujours franche: d'une part, il arrive qu'un indicateur puisse être rangé tantôt dans une catégorie, tantôt dans une autre; d'autre part, la corrélation entre moyens mis en œuvre et résultat obtenu est parfois malaisée à établir: seuls les professionnels de la fonction $\mathrm{RH}$ peuvent tenter d'élaborer des indicateurs corrélant les faits générateurs et les effets désirés.

Le tableau de bord, quant à lui, réunit cycliquement un ensemble d'indicateurs désignés comme étant les principaux leviers d'action. Il peut être de deus types : simple ou complexe. Le premier privilégie le lien entre critères de performance et objectifs; il permet un suivi de l'activité et de ses performances. Le second intègre l'usage des critères de réalisation et permet, par conséquent, un suivi de la conformité et/ou de la pertinence du processus luimême. En tout état de cause, le tableau de bord ne doit comporter que les indicateurs que l'on souhaite particulièrement surveiller. Il doit, en outre, être cohérent et non construit comme l'assemblage d'une mosaïque d'indicateurs disparates. Il doit, enfin, permettre des comparaisons dans la mesure où un indicateur seul est vide de sens. Il peut ainsi être placé dans un référentiel de temps (plus les comparaisons au passé sont nombreuses, plus les projections sont crédibles) et rapproché de normes externes à l'entreprise, telles que : la moyenne nationale, internationale (Europe et hors Europe) et/ou celle de la branche (par taille d'entreprise, types de produits fabriqués, catégorie de personnel, etc). 


\section{Indicateurs suggérés par les textes de référence}

Que nous indiquent les textes de référence au sujet de ces indicateurs? L'Afnor donne assez peu d'indications sur la mesure de la diversité pouvant permettre l'obtention du Label Diversité: "L'organisme doit définir et mettre en cuvre des outils adaptés pour mesurer la diversité quand cela est possible, pour analyser ses pratiques, pour identifier les plaintes (réclamations) internes ou externes provenant de remontée directes et/ou via les partenaires sociaux, des entretiens 'évaluation, des collaborateurs quittant l'organisme. Ces outils doivent garantir la confidentialité et/ou l'anonymat. L'organisme doit évaluer la satisfaction de son personnel en matière de diversité. »(Label Diversité, Afnor, Domaine 5 : Evaluation et axes d'amélioration de la politique diversité).

En revanche, l'Afnor est beaucoup plus prolixe en matière de recommandations d'organisation et de pilotage en ce qui concerne la Norme Diversité, et donne de nombreux exemples d'indicateurs à ce niveau (pour la liste complète se référer aux annexes). Ses exigences se situent à sept niveaux, dont la dernière renvoie, précisément, à l'exigence de mesurer les résultats obtenus en matière de diversité :

- l'engagement de l'organisme (par exemple, la création d'une instance interne dédiée)

- l'implication des partenaires sociaux (par exemple, l'association de représentants du personnel et/ou d'organisations syndicales à la politique de diversité

- la communication interne (par exemple, la valorisation d'actions permettant de faire reculer les pratiques discriminatoires)

- la sensibilisation et à la formation à la politique diversité (il s'agit de produire le programme de formation relatif à diverses populations telles le Comité de direction ou les organes décisionnels équivalents)

- le processus de Ressources Humaines (par exemple, l'implication du management qui peut être mesurée à travers les comptes-rendus de réunion de calibrage des évaluations des Managers

- les relations avec les territoires (par exemple, l'évaluation de l'impact des partenariats à travers les coupures de presse)

- l'évaluation des résultats de la politique Diversité sur la base d'outils de mesure permettant d'obtenir une photographie de l'organisme à un instant donné et d'appréhender les progrès. 
Par ailleurs, le rapport social donne également des directives sur la mesure de la diversité. Sur les 35 indicateurs obligatoires devant figurer dans le rapport, 5 sont en lien direct avec la diversité au sens étroit ou large : effectifs hommes et femmes, effectifs handicapés, participants aux actions de formation des hommes et des femmes, rémunération moyenne annuelle brute des hommes et des femmes et, enfin, effectif en contrat d'alternance (Novethic, 2005). Pour chacun de ces cinq indicateurs, nous mentionnerons deux composantes, à titre illustratif :

- Approche globale de la diversité

$\checkmark$ Le président mentionne-t-il l'enjeu diversité dans son message ?

$\checkmark$ Y a-t-il une photo permettant d'évaluer la diversité de l'exécutif?

- Diversité liée au genre

$\checkmark$ Taux de femmes dans l'encadrement/management

$\checkmark$ Existence d'un accord cadre ou d'entreprise

- Diversité liée à l'âge

$\checkmark$ Mention de l'âge moyen des salariés

$\checkmark$ Mention de la répartition des effectifs par âges

- Diversité liée au handicap

$\checkmark$ Taux de salariés handicapés dans l'effectif

$\checkmark$ Indicateurs sur le recrutement de travailleurs handicapés

- Diversité liée à l'origine

$\checkmark$ Mention sur la nationalité des salariés

$\checkmark$ Mention de plaintes pour cause de discrimination (au niveau Groupe)

\section{Les tableaux de bord de la Diversité : enjeux communicationnels et stratégiques}

Tout d'abord le tableau de bord renvoie à une tactique communicationnelle dans la mesure où les indicateurs peuvent revêtir différentes formes. Selon Feisthammel et Massot (2005), «Tous les supports d'expression peuvent a priori servir de support de présentation pour restituer l'information significative d'un indicateur ». Citons, de façon non exhaustive, du plus «brut » au plus « figuratif» (avec, par conséquent, un besoin d'interprétation décroissant et une signification croissante): statistiques, ratios, pourcentages bruts, pourcentages par tranches, calculs (moyenne, écart-type, médiane...), affectation de couleurs, représentations graphiques (par exemple : :) ou $:)$ ), réponse à une question (sous forme de mots ou sous 
forme binaire : oui, non), commentaire, interpellation (un des DRH que nous avons interrogé, a lancé durant une réunion d'un Comité de direction : "On prône la diversité, mais il n'y a pas une seule femme ici présente! »), etc.

Comme on le voit, les supports d'expression pouvant être utilisés dans la cadre de la diversité sont nombreux, allant du quantitatif au qualitatif, du nominal au verbal. Ils peuvent faire appel à l'hémisphère droit du cerveau (chiffres, mots) ou à l'hémisphère gauche (dessin, couleur). Le manager de la diversité peut avoir tout à la fois intérêt à afficher à l'extérieur un équilibre entre le nombre de femmes et d'hommes présents dans l'entreprise afin d'attirer un vivier de femmes à haut potentiel et alerter en interne sur l'insuffisance de femmes à des postes de direction ; il peut compiler des indicateurs de nature différente - femmes et seniors- afin de dramatiser le risque de discrimination ; pour contourner une difficulté à collecter de grandes masses de données qui lui seraient pourtant utiles, il peut proposer un indicateur qualitatif sous forme de perceptions -sur la base d'interviews, par exemple- d'acteurs clé de l'entreprise sur le sujet; il peut avoir recours à des langages ne suscitant pas d'interprétation (tels que l'utilisation de couleurs) lorsqu'il a besoin d'attirer l'attention et d'encourager une lisibilité immédiate.

En seconde lieu, le tableau de bord renvoie aussi à des aspects stratégiques. Contrairement aux démarches de lutte contre les discriminations, celles qui favorisent la diversité revêtent un caractère volontaire: leur succès dépend donc, entre autres, des stratégies de persuasion développées pas les responsables de projets (que nous nommerons dorénavant Managers de la Diversité). Ainsi que le suggère Landrieux-Kartochian (2007), ces acteurs sont en quête permanente de légitimité et s'apparentent aux «champions» de l'innovation organisationnelle dans le sens où leur rôle est de contribuer de façon déterminante à un projet « en promouvant activement et avec enthousiasme son avancée au cours des phases critiques de manière à obtenir des ressources et/ou un soutien actif du top management » (Grima et Trépo, 2003, p. 24). En cela, il doit se battre sur trois fronts : obtenir le soutien des dirigeants pour garantir les ressources permettant de mener à bien le projet, convaincre les partenaires du projet, sur la base d'argument rationnels, de l'impact de la diversité sur la performance de l'entreprise -argument dénommé business case- et, enfin, développer des motifs légitimes par rapport à la culture de l'entreprise. 


\section{Difficultés rencontrées par les entreprises et choix effectués en matière d'indicateurs}

\section{Difficultés rencontrées}

Nous avons vu qu'il n'est pas toujours aisé de déterminer quels indicateurs suivre. Une fois ce travail effectué, encore faut-il aller à la pêche des informations requises. Dès nos premiers entretiens, il est très clairement apparu que les systèmes d'informations (SI) des entreprises n'ont pas été conçus dans le but d'obtenir des informations sur la diversité. Même si certaines informations recherchées se trouvent dans des rapports obligatoires comme la situation comparée femme-homme ou le bilan social, chaque entreprise a été obligée de monter des systèmes ad hoc spécifiquement pour cette problématique. Toutes les entreprises avec qui nous avons parlé ont passé beaucoup de temps à collecter les informations.

Une autre difficulté réside dans la définition même des termes, qui a évidemment une incidence sur les indicateurs. Par exemple, si une entreprise veut travailler sur une politique à destination des seniors, la première chose à faire est de définir le terme seniors. Malgré l'existence de certaines définitions dans des textes officiels, l'exercice n'est pas facile.

Une fois décidé quels indicateurs une entreprise veut suivre, elle n'est pas toujours en mesure de les comparer à d'autres entreprises, en France ou à l'international, parce qu'elle n'utilise pas nécessairement la même définition que d'autres entreprises mais aussi tout simplement parce que peu d'entreprises divulguent leurs chiffres.

Cela nous amène à sans doute la plus grande difficulté rencontrée par les entreprises, à savoir le manque de recul et de maturité sur le sujet:

«C'est un peu pour nous l'année zéro du développement des indicateurs propres à la diversité, même si, l'an dernier, on a déjà amorcé un peu les choses. »

«Là, on est vraiment à un tournant. (...) avec l'audit qu'on a fait et les autres améliorations qui vont forcément nous faire ouvrir de nouveaux indicateurs, de nouveaux suivis.»

\section{Quel type d'indicateurs utilisent les entreprises?}


Au vu des choix effectués par les entreprises de notre panel, nous observons tout d'abord que la grande majorité d'entre elles se concentre sur trois dimensions de la diversité : l'âge, le genre et le handicap. En effet, ces dimensions ne posent pas de problème particulier de mesure, ont les dispositifs réglementaires les plus développés ou sont rattachés à une incitation financière (nous pensons en particulier au handicap). Seules certaines vont plus loin et tentent aussi de mesurer les origines (sociales, scolaires, géographiques et/ou ethniques)

Nous constatons aussi que l'usage qui est fait des indicateurs est assez différencié d'une entreprise à l'autre: destination interne et/ou externe, usage à des fins de communication, d'action, d'obtention d'un label, utilisation passive (répertorier la diversité, logique de suivi) ou active (piloter la diversité, logique de changement), hiérarchisation faite entre différentes dimensions de la diversité ou non, etc. Par exemple, une des entreprises de notre panel ne fait figurer dans le tableau de bord du Président que deux indicateurs - le pourcentage de femmes appartenant au comité de Direction et de personnes handicapées - tandis que d'autre indicateurs sont utilisés par le reste de l'entreprise : âge, personnes issues des DOM ; personnes issues des zones sensibles (ZUS). Certaines entreprises s'appuient sur les indicateurs retenus mais n'hésitent pas à «faire feu de tout bois » en interpellant, par exemple, sur le fait qu'il y ait une majorité d'hommes dans une réunion d'encadrement. Les unes s'appuient essentiellement sur les indicateurs rendus obligatoires par les textes (protocole GRI, rapport de situation comparée, accords de branche, etc.) tandis que les autres en construisent également de façon ad hoc. Certaines entreprises avancent sur plusieurs fronts en même temps tandis que d'autres préfèrent progresser rapidement sur l'un de ces fronts (généralement, le handicap, l'âge et/ou le genre), en collaboration avec les syndicats, avant de développer les autres. De manière générale, les entreprises nous disent tâtonner et en être à leurs tous débuts en matière de réflexion et pratiques sur ce sujet.

Mais quels indicateurs les entreprises de notre échantillon ont-elles retenus ? On observe plusieurs éléments : il s'agit généralement de données brutes - c'est-à-dire qu'ils ne sont ni croisés avec une dimension de la diversité ni rapportés à une population cible, ce qui en réduit l'intérêt; ce sont des indicateurs qui décrivent davantage la non-discrimination que la diversité; il s'agit, le plus souvent, d'indicateurs de résultat et, en sous-catégorie, d'indicateurs de structure; enfin, la totalité des indicateurs sont des indicateurs d'équilibration. Nous avons sélectionné ci-dessous les indicateurs qui nous ont été fournis par les entreprises de notre échantillon qui nous semblent les plus intéressants (au sens où ils 
mélangent indicateurs de résultat, de structure, d'équilibration, de diversité et de nondiscrimination) :

\begin{tabular}{|c|c|c|c|}
\hline \multirow[b]{2}{*}{ Exemples d'indicateurs } & \multirow[b]{2}{*}{ Enjeu } & \multicolumn{2}{|c|}{$\begin{array}{l}\text { Caractéristiques des } \\
\text { indicateurs construits }\end{array}$} \\
\hline & & $\begin{array}{c}\text { Type } \\
\text { d'indicateur } \\
\text { s }\end{array}$ & $\begin{array}{l}\text { Nature des } \\
\text { indicateurs }\end{array}$ \\
\hline Répartition salariés H/F promus par classification & $\begin{array}{c}\text { Non- } \\
\text { Discrimination }\end{array}$ & $\begin{array}{c}\text { Résultat } \\
\text { (performance } \\
\text { ) }\end{array}$ & Equilibration \\
\hline Nombre de congés de paternité & Diversité & $\begin{array}{l}\text { Résultat } \\
\text { (structure) }\end{array}$ & Equilibration \\
\hline $\begin{array}{l}\text { Nombre de candidatures reçues par l'entreprise } \\
\text { dans l'année : répartition par âge }(+45 \text { ans et }+55 \\
\text { ans) }\end{array}$ & $\begin{array}{c}\text { Non- } \\
\text { Discrimination }\end{array}$ & $\begin{array}{c}\text { Résultat } \\
\text { (performance } \\
\text { ) }\end{array}$ & Equilibration \\
\hline $\begin{array}{l}\text { Embauches de l'année : répartition par niveau de } \\
\text { diplôme et par âge ( }+45 \text { ans et }+55 \text { ans) }\end{array}$ & Diversité & $\begin{array}{l}\text { Résultat } \\
\text { (performance } \\
\text { ) }\end{array}$ & Equilibration \\
\hline $\begin{array}{l}\text { Nombre de salariés de plus de } 45 \text { et } 55 \text { ans } \\
\text { promus dans une catégorie supérieure (avec une } \\
\text { répartition par âge) }\end{array}$ & $\begin{array}{c}\text { Non- } \\
\text { Discrimination }\end{array}$ & $\begin{array}{c}\text { Résultat } \\
\text { (performance } \\
\text { ) }\end{array}$ & Equilibration \\
\hline $\begin{array}{l}\text { Politique de recrutement spécifique pour les } \\
\text { femmes }\end{array}$ & $\begin{array}{l}\text { Egalité des } \\
\text { Chances }\end{array}$ & $\begin{array}{l}\text { Résultat } \\
\text { (performance }\end{array}$ & Equilibration \\
\hline $\begin{array}{l}\text { Existence d'un système de "mentor" pour les } \\
\text { hauts potentiels femmes }\end{array}$ & $\begin{array}{l}\text { Egalité des } \\
\text { Chances }\end{array}$ & Moyen & Equilibration \\
\hline $\begin{array}{l}\text { Programme pour équilibrer vie personnelle et vie } \\
\text { professionnelle }\end{array}$ & $\begin{array}{l}\text { Egalité des } \\
\text { Chances }\end{array}$ & $\begin{array}{l}\text { Résultat } \\
\text { (structure) }\end{array}$ & Equilibration \\
\hline Nombre de contrats en alternance conclus & $\begin{array}{l}\text { Egalité des } \\
\text { Chances }\end{array}$ & $\begin{array}{c}\text { Résultat } \\
\text { (performance } \\
\text { ) }\end{array}$ & Equilibration \\
\hline Réalisation d'un « testing » des « process » RH & $\begin{array}{c}\text { Non- } \\
\text { Discrimination }\end{array}$ & $\begin{array}{c}\text { Résultat } \\
\text { (performance } \\
\text { ) }\end{array}$ & Equilibration \\
\hline Message fort du Comex en faveur de la diversité & $\begin{array}{c}\text { Non- } \\
\text { Discrimination }\end{array}$ & Moyen & Equilibration \\
\hline $\begin{array}{l}\text { Testing de recrutement (par l'Observatoire des } \\
\text { Discriminations ou autres) }\end{array}$ & $\begin{array}{l}\text { Egalité des } \\
\text { Chances }\end{array}$ & $\begin{array}{c}\text { Résultat } \\
\text { (performance }\end{array}$ & Equilibration \\
\hline $\begin{array}{l}\text { Politique de recrutement spécifique pour les } \\
\text { femmes }\end{array}$ & $\begin{array}{l}\text { Egalité des } \\
\text { Chances }\end{array}$ & Moyen & Equilibration \\
\hline Programmes d'accompagnement type tutorat & $\begin{array}{c}\text { Non- } \\
\text { Discrimination }\end{array}$ & Moyen & Equilibration \\
\hline Nombre de contrats en alternance conclus & $\begin{array}{l}\text { Egalité des } \\
\text { Chances }\end{array}$ & $\begin{array}{l}\text { Résultat } \\
\text { (structure) }\end{array}$ & Equilibration \\
\hline $\begin{array}{l}\text { Annonces / Publireportages dans des supports } \\
\text { ciblés pour les personnes handicapées }\end{array}$ & $\begin{array}{c}\text { Non- } \\
\text { Discrimination }\end{array}$ & Moyen & Equilibration \\
\hline $\begin{array}{l}\text { Chiffre d'affaires réalisé avec le secteur protégé } \\
\text { par salarié }\end{array}$ & $\begin{array}{c}\text { Egalité des } \\
\text { Chances }\end{array}$ & Moyen & Equilibration \\
\hline
\end{tabular}




\section{Propositions et pistes d'amélioration}

Nous souhaitons présenter deux classifications proposées par la revue de littérature en matière de diversité dans la mesure où elles peuvent nous aider à qualifier les indicateurs choisis par les entreprises (pertinence substantielle, couverture, précision...).

La première classification renvoie aux aspects stables et instables, visibles et non visibles de la diversité (Falcoz et Barth 2007) :

\begin{tabular}{|l|l|l|}
\hline & Différence visible & Différence non visible \\
\hline Différence stable & $\begin{array}{l}\text { Genre, } \\
\text { Origine ethnique }\end{array}$ & $\begin{array}{l}\text { Orientation sexuelle, } \\
\text { Certains handicaps }\end{array}$ \\
\hline Différence instable & $\begin{array}{l}\text { Age, } \\
\text { Activités syndicales }\end{array}$ & $\begin{array}{l}\text { Certains handicaps, } \\
\text { Convictions religieuses }\end{array}$ \\
\hline
\end{tabular}

La seconde classification embrasse une définition plus large de la diversité puisqu'elle distingue les cinq caractéristiques suivantes de la diversité (Cornet et Delahaye, 2006): les caractéristiques physiques «visibles », les caractéristiques physiques «fonctionnelles » (ce qui peut handicaper / exclure), les « autres caractéristiques individuelles relevant de l'histoire de vie de l'individu, les caractéristiques sociales (appartenance réelle ou supposée à un groupe ou un environnement social) et, enfin, les caractéristiques organisationnelles (renvoie à l'appartenance à un métier ou une organisation).

\begin{tabular}{|c|c|c|c|c|}
\hline $\begin{array}{c}\text { Caractéristique } \\
\text { s physiques } \\
\text { visibles }\end{array}$ & $\begin{array}{l}\text { Caractéristique } \\
\text { s physiques } \\
\text { fonctionnelles }\end{array}$ & $\begin{array}{c}\text { Caractéristique } \\
\text { s individuelles } \\
\text { liées à l'histoire } \\
\text { de vie de } \\
\text { l'individu } \\
\end{array}$ & $\begin{array}{c}\text { Caractéristiques } \\
\text { sociales }\end{array}$ & $\begin{array}{c}\text { Caractéristiq } \\
\text { ues } \\
\text { organisationn } \\
\text { elles }\end{array}$ \\
\hline $\begin{array}{c}\text { Âge } \\
\text { Origine ethnique } \\
\text { et couleur de } \\
\text { peau } \\
\text { Sexe } \\
\text { Orientation } \\
\text { sexuelle } \\
\text { Taille } \\
\text { Poids }\end{array}$ & $\begin{array}{c}\text { Handicap } \\
\text { physique } \\
\text { Handicap } \\
\text { mental } \\
\text { Taille } \\
\text { Poids } \\
\text { État de santé } \\
\text { (grossesse, } \\
\text { maladies } \\
\text { chroniques...) }\end{array}$ & $\begin{array}{c}\text { Ex-détenus et/ou } \\
\text { dossier } \\
\text { judiciaire } \\
\text { Degré de } \\
\text { maîtrise de } \\
\text { certaines } \\
\text { langues } \\
\text { Expérience } \\
\text { professionnelle } \\
\text { Expérience de } \\
\text { bi-culturalité } \\
\text { Orientation } \\
\text { sexuelle }\end{array}$ & $\begin{array}{c}\text { Nom et prénom } \\
\text { Langue maternelle } \\
\text { Religion } \\
\text { Situation familiale } \\
\text { Origine sociale et classe } \\
\text { sociale } \\
\text { Communauté culturelle } \\
\text { et/ou linguistique } \\
\text { Lieu de vie } \\
\text { Orientations politiques } \\
\text { Nationalité } \\
\text { Parcours professionnel }\end{array}$ & $\begin{array}{c}\text { Métiers ou } \\
\text { professions } \\
\text { Départements } \\
\text { ou services }\end{array}$ \\
\hline
\end{tabular}

De notre point de vue, ces classifications sont susceptibles de guider les Managers de la diversité dans leur élaboration d'indicateurs qui reflètent la réalité autant qu'ils la 
construisent. A titre d'exemple, la pertinence substantielle des indicateurs - leur degré de fidélité à la situation évaluée - est plus forte pour la diversité visible que pour la diversité non-visible (telle que certains handicaps) dans la mesure où une certaine proportion de nondéclaration qui fausse la fiabilité de l'indicateur. De même, les décisions relatives à la temporalité et à la fréquence de la mesure seront différentes selon que la diversité est stable ou non. La conformité politique - le degré de cohérence entre la nature de l'indicateur retenu et la politique d'activité souhaitée par les décideurs - sera plus grande si les indicateurs relatifs aux caractéristiques physiques, d'une part et sociales, d'autre part, sont utilisés dans une logique d'égalité de traitement et que les indicateurs relatifs à l'histoire de vie et plus largement, à la diversité dite «profonde », sont exploités dans une logique d'égalité des chances et, enfin, si les caractéristiques organisationnelles sont employées à servir une et politique Diversité à proprement parler.

Pour notre part, nous proposons une classification qui permet d'intégrer les termes utilisés par les entreprises en France aujourd'hui :

- égalité de traitement (logique juridique): évitement des risques pénaux (et d'image) associés à la discrimination

- égalité des chances (logique sociale): légitimité, reflet de bassin d'emploi ; RSE, politique RH -attractivité, motivation, fidélisation...

- diversité (logique économique) : pénurie de talents ; innovation, créativité, apprentissage organisationnel

De notre point de vue, l'égalité de traitement est enchâssée par l'égalité des chances, qui est elle-même enchâssée par la diversité.

\section{Conclusion}

Nous conclurons ce texte sur plusieurs idées. Tout d'abord, l'indicateur dans le domaine de la diversité constitue à la fois une contrainte (réponse à une obligation légale) et une opportunité (levier d'action pour faire reculer les inégalités, amélioration), à des dosages variables selon le degré de maturité de l'organisation. Ensuite, la construction des indicateurs et des tableaux de bord répond à des enjeux tant opérationnels que pédagogiques, stratégiques ou communicationnels. En ce sens, il faut distinguer les indicateurs sur lesquels l'organisation communique vers l'extérieur, ceux qu'elle suit en interne, ceux qu'elle diffuse à la Direction Générale, dont le soutien au projet est indispensable- l'indicateur constitue un moyen et non 
une fin. De fait, une agilité dans l'utilisation des indicateurs et une bonne intelligence de l'outil sont requises : répondre aux questions «pour quoi, pour qui, quand mesurer?» constitue un pré-requis minimal. En outre, un tableau de bord de la diversité doit, idéalement, à la fois comporter des indicateurs de moyen et de résultat, de structure et de performance, d'alerte, d'équilibration et d'anticipation. Or, les entreprises ne sont emparés du sujet des indicateurs que très récemment, n'ont généralement pas encore fixé d'objectifs ou de seuils en matière d'égalité de chances, ne disposent la plupart du temps pas de systèmes d'information adaptés aux besoins de la diversité et donc rares sont-elles qui ont une palette d'indicateurs répondant à ces exigences. Enfin, développer une politique active en faveur de la diversité ne constitue que le premier pas d'un parcours délicat mais passionnant : celui de l'intégration des différences dans le but de produire un effet générateur du type «1+1=3». Encore reste-t-il à comprendre les conditions requises dans le contexte particulier de chaque entreprise permettant à la diversité d'être source de créativité, d'innovation et, in fine, de performance socio-économique.

\section{Bibliographie}

Falcoz C, et Barth I. (2007), Le management de la diversité : enjeux, fondements et pratiques », L'Harmattan.

Feisthammel D. et Massot P. (2005), Fondamentaux du pilotage de la performance. Le tableau de bord adapté à la réalité des activités, Paris : AFNOR.

Fernandez A. (2005), Les nouveaux tableaux de bord des managers, Paris: Editions d'Organisation.

Grima F. et Trépo G. (2003), «Initier une innovation organisationnelle : tactiques d'influence et processus de persuasion mis en œuvre par les champions », Revue de Gestion des Ressources Humaines, Vol. 50, octobre-décembre, p. 23-36.

Landrieux-Kartochian S. (2007), «Les organisations face au plafond de verre », Revue Française de Gestion, № 173, p. 15-30.

McKinsey \& Company, « Woman matter », 2007.

Taïeb J.P. (1996), Les tableaux de bord de la gestion sociale, Dunod, Paris. 